\title{
Seroprevalence of Measles Antibodies among Young Adults in a Tertiary Care Hospital, India
}

\author{
L. Prashanthi, N. Sridevi*, Dara Saranya and P. Sashikala Reddy \\ Department of Microbiology, Osmania Medical College, Hyderabad, India \\ *Corresponding author
}

\begin{abstract}
Keywords
Measles,

Antibodies, Adults, IgG ELISA Kit significantly increased. Many countries have reported diminishing of antibody titres against measles among young population as immunization coverage of adolescents and adults in not monitored. Objective is to determine seroprevalence of $\mathrm{IgG}$ antibodies titres against measles in young adults. It is a cross sectional study, conducted over 6 months (JUNE to NOVEMBER 2018) Sample size is 200. Study population belonged to 15-30 yrs of age. Samples - serum (Blood) Measles IgG ELISA Kit (EUROIMMUN). Out of the 200 samples collected, $65.6 \%$ had protective titres, $21.9 \%$ of the study group showed susceptibility i.e. they had unprotective levels of antibody titres and $12.5 \%$ were in the borderline titre group. Most of the individuals were vaccinated with a single dose of measles vaccine. Several reports have shown that the susceptibility to measles infection may be rising because of waning vaccine induced immunity over time after vaccination, in the absence of natural boosting by circulating measles viruses While most of the immunization drives in developing countries are aimed at children, it is important to recognize and identify pockets of susceptible populations that could jeopardize the prevention and control of these vaccine preventable diseases. Hence there is a need for serosurveillance and mop -up programs to cover these pockets of susceptible population so as to reach the WHO goal of Measles Eradication by 2020.
\end{abstract}

\section{A B S T R A C T}

Measles is a highly contagious vaccine-preventable disease caused by the Morbilivirus. Measles vaccine was introduced in India in 1985 as a part of Universal Immunization Programme (UIP) which was given at 9 completed months of age. As vaccine coverage has increased, there has been a marked reduction in measles incidence in the pediatric age group; but at the same time, the average age, at which measles infection occurs, has

\section{Introduction}

Measles is a highly contagious vaccinepreventable disease caused by the Morbilivirus. It is a single-stranded, negativesense, enveloped RNA, which belongs to the family Paramyxoviridae. Measles is characterized by the presence of fever, cough, and coryza, followed by the appearance of a typical rash (9).

Measles vaccine was introduced in India in 1985 as a part of Universal Immunization Programme (UIP) which was given at 9 
completed months of age. Two-dose vaccination strategy was introduced in India in 2010 (19)

As vaccine coverage has increased, there has been a marked reduction in measles incidence in the pediatric age group; but at the same time, the average age, at which measles infection occurs, has significantly increased (10)

In spite of the availability of vaccine, more than 20 million measles cases are reported every year all over the globe. In 2010, alone 139,300 deaths due to measles were reported underlining the public health importance of this infection (13).

Although most reported measles cases are still in young and school-aged children, recent cases and outbreaks have also occurred in isolated communities of adults. Approximately $25 \%$ of the cases reported in 2008 were in people age 20 and older.

Study findings have indicated that more than $50 \%$ of the global measles associated deaths were reported in India alone (19) Measles outbreaks among highly vaccinated populations have been observed in many countries $(15,9,19)$ Recently, three outbreaks of measles in adults (medical cadets, medical students and nursing students) were reported from the State of Maharashtra, Karnataka and Andhra Pradesh. It is clear that Measles is a re-emerging disease.

Vaccine, the most important intervention in the transmission of measles, is failing to confer enough immunity to public and is evident by the fact that alarming number of cases in previously vaccinated subjects have been observed (11).

Many countries have reported diminishing of antibody titres against measles among young population as immunization coverage of adolescents and adults in not monitored.

These outbreaks of measles among young population emphasize the need for serosurveillance of susceptible population and strengthening of vaccine coverage. In view of the present scenario of measles in India, the aim of this study was to determine the susceptibility against measles among young adults.

\section{Materials and Methods}

\section{Study area and population}

The study population included young adults (15-30 yrs) and was carried out in Microbiology laboratory of Osmania General Hospital, a tertiary care teaching institute over a period of 6 months from July- December 2018.

\section{Sample collection and size}

$5 \mathrm{ml}$ of whole blood sample was collected in a serum separator vacutainer under aseptic conditions from 200 healthy individuals within the age group of 15-30 years. The serum was separated and stored at $-2^{\circ} \mathrm{C}$.

\section{Test Details}

Detailed history of the individual was taken regarding immunization status. The presence of specific IgG antibodies against measles in the obtained sera samples was determined by the ELISA method. We used the commercial EUROIMMUN Measles Virus IgG KIT. The test was performed according to the instruction booklet of manufacturer.

\section{Evaluation of results}

The results were evaluated qualitatively as positive, negative and borderline. Positive 
results were evaluated as corresponding to $200 \mathrm{IU} / \mathrm{ml}$ and over, negative under 200 $\mathrm{IU} / \mathrm{ml}$ and borderline 201-275 IU/ml titres.

\section{Results and Discussion}

Out of 200 individuals who participated in the study, according to the vaccination history given $182(91 \%)$ were vaccinated, 8 (4\%) were not vaccinated and $10(5 \%)$ of their vaccination status was unknown (Figure 1). Of the total samples obtained, all the individuals belonged to the age between 1530 years. Individuals were categorized into 3 age groups, 30 of them belonged to 15-20 yrs (years of birth 1999-2004),100 belonged to 21-25yrs (years of birth 1994-1998), 62 belonged to 26-30 yrs (years of birth 19891993). Age wise distribution amongst these individuals showed most of them belonging to 21-26 years, followed by 26-30 years (Figure 2).

Vaccination against measles in India was introduced in 1985 in a one-dose schedule, which was changed to a two-dose schedule in 2010. It means all the study population of the 3 categories of age was vaccinated only with one dose of measles. In 200 serum samples obtained, 94 of them were males (47\%) and $102(51 \%)$ were females. There was almost equal gender representation (Table 1).

Based on the commercial kit, titre value percentages of population having negative titre, positive titre and borderline titres were as follows (Figure 3). International value 200 IU/L is seen as a correlate of protection. $21.9 \%$ of population have negative titre values, $12.5 \%$ of population have borderline titres $(12.5 \%)$ and $65.6 \%$ have protective titres $(65.6 \%)$. The study also shows that most of the individuals having protective titres were in the age group of 15-20 years. Majority of the people in 21-26 years' age group had borderline titres. Almost all of them in the age group of 26-30 years had unprotective antibody titres (Figure 4 and 5).

Despite high measles vaccination coverage by a successful national vaccination program, many outbreaks have occurred among vaccinated young adults. (12) In the present study the recorded overall seropositivity of the specific IgG antibodies against measles as $65.6 \%, 21.9 \%$ of the tested samples were seronegative and $12.5 \%$ were borderline titres.

Similar susceptibility or seronegative titres was observed by Gomber et al., (21.4\%) and Raut et al (19.2\%). The lowest seropositivity i.e. unprotective titres of measles $\operatorname{IgG}$ antibodies in current study were detected in the age group of 26-30 years. This age group was formed by vaccinated persons, but they were given only one dose of vaccine during the first years of vaccination implementation into the Universal immunization program. This corresponds to the study findings of Trmal et al., (18).

The second lowest seropositivity or borderline antibody titres was detected in the age group of 21-25 years. The highest seropositivity or protective levels of $\mathrm{IgG}$ antibodies was detected in the age group of 15-20 years.

Several reports have warned that the susceptibility to measles infection may be rising because of waning vaccine induced immunity over time after vaccination, in the absence of natural boosting by circulating measles viruses. (8). In fact, it has been shown that the levels of antibodies developed in response to the vaccine decline at a much faster rate than when naturally acquired (4b)Simultaneously, an increase of seronegative individuals among the vaccines from the time of introduction of measles vaccination can be observed in present study. 
Table.1

\begin{tabular}{|l|l|l|}
\hline AGE GROUP & MALES & FEMALES \\
\hline $15-20$ YRS & 16 & 18 \\
\hline $21-25$ YRS & 40 & 60 \\
\hline $26-30$ YRS & 38 & 24 \\
\hline
\end{tabular}

Fig.1

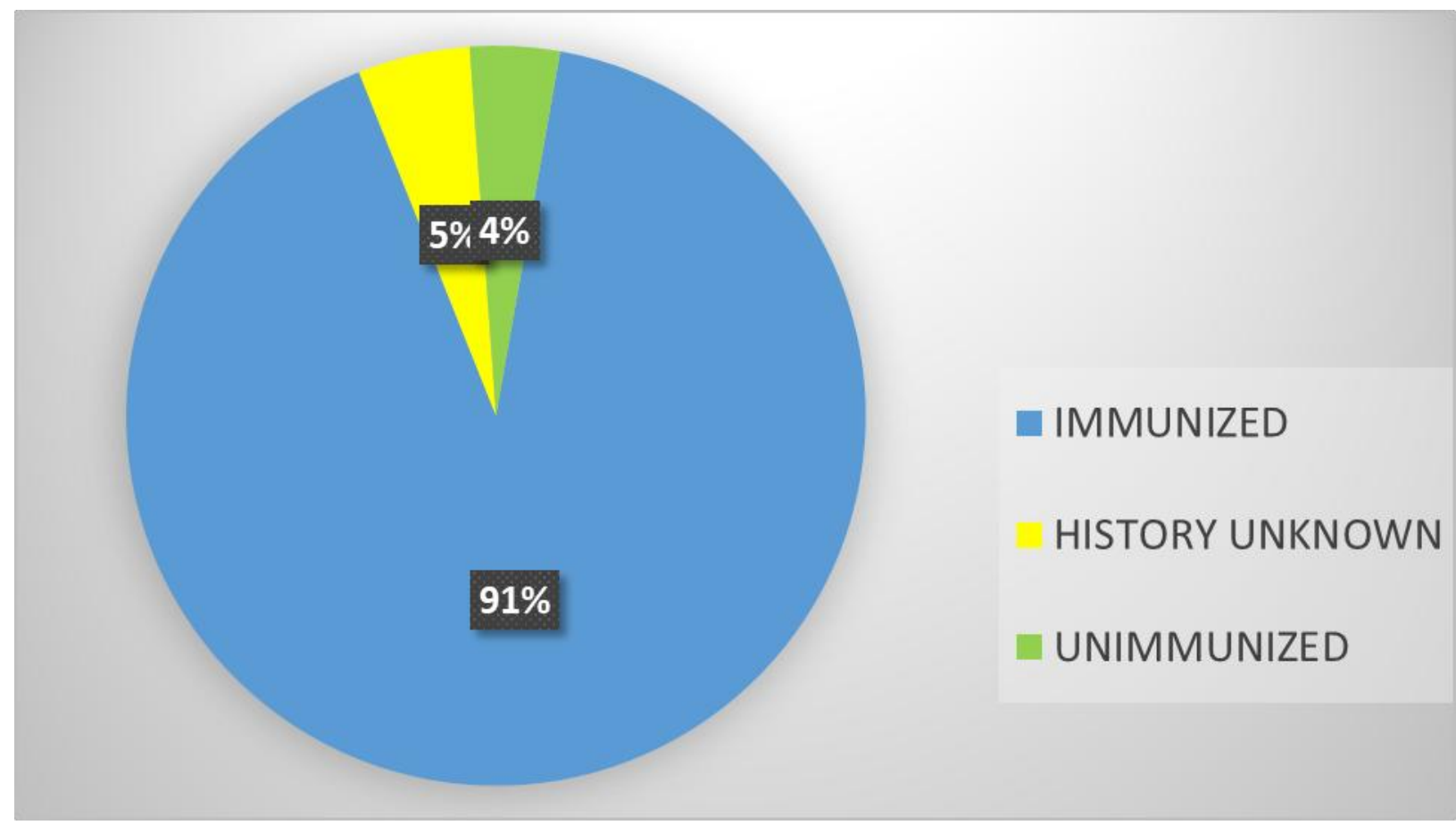

Fig.2

\section{NUMBER OF PEOPLE IN EACH GROUP}

\section{0}

100

80

60

40

20

0

$$
30
$$

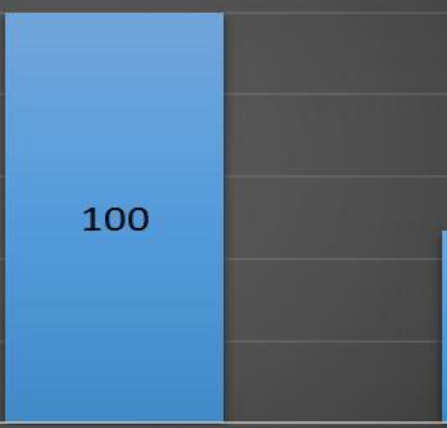


Fig.3

\section{PROTECTIVE TITRES PERCENTAGE}

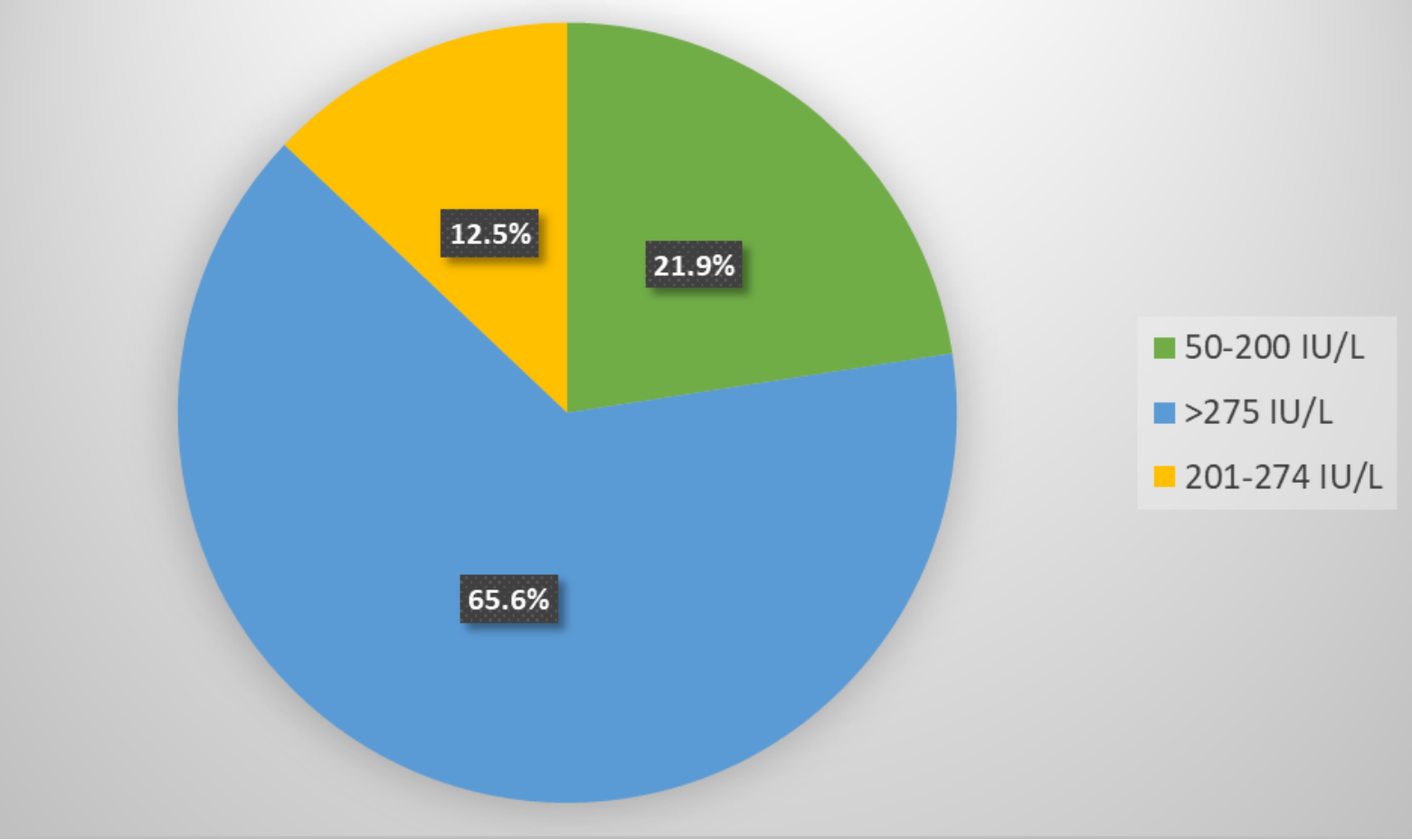

Fig.4

Age Wise Distribution

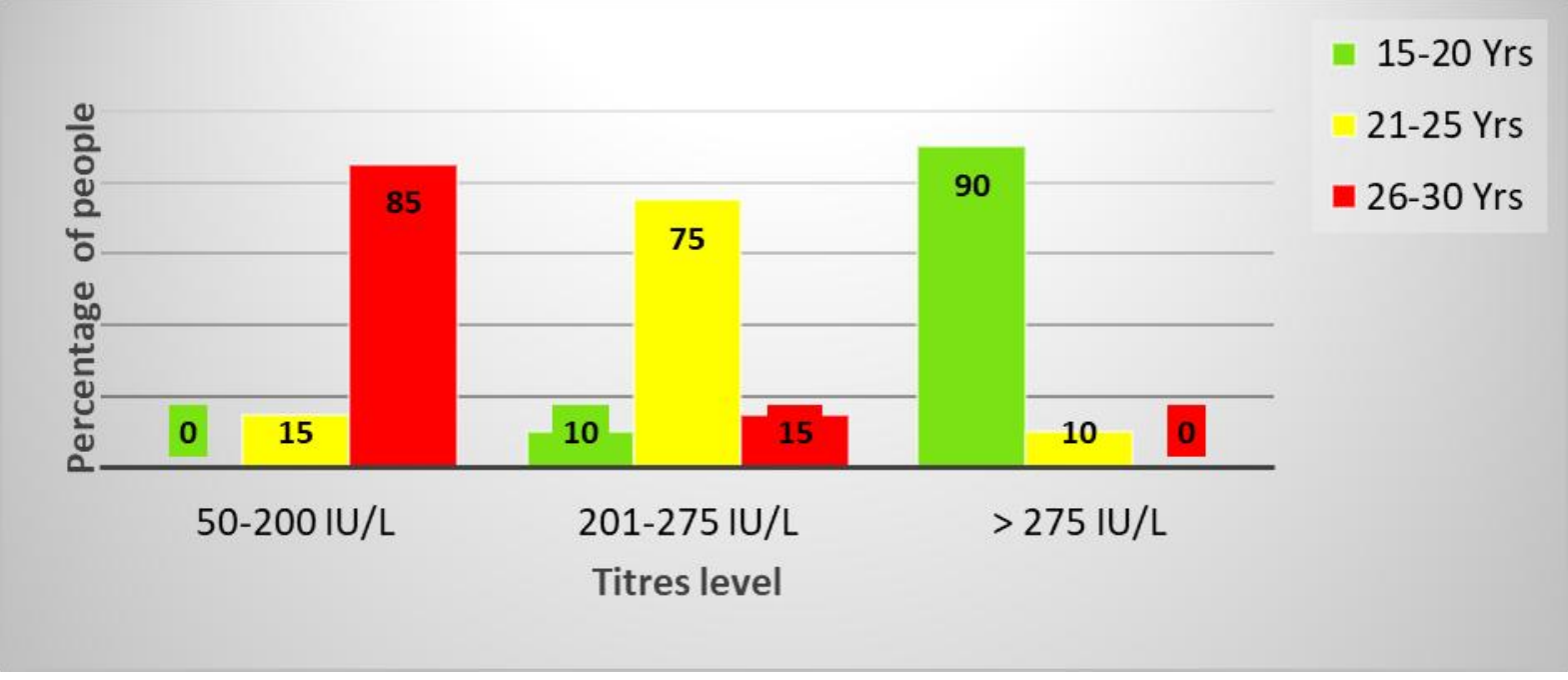


Fig.5

\section{POPULATION AT RISK DUE TO WANING TITRES}

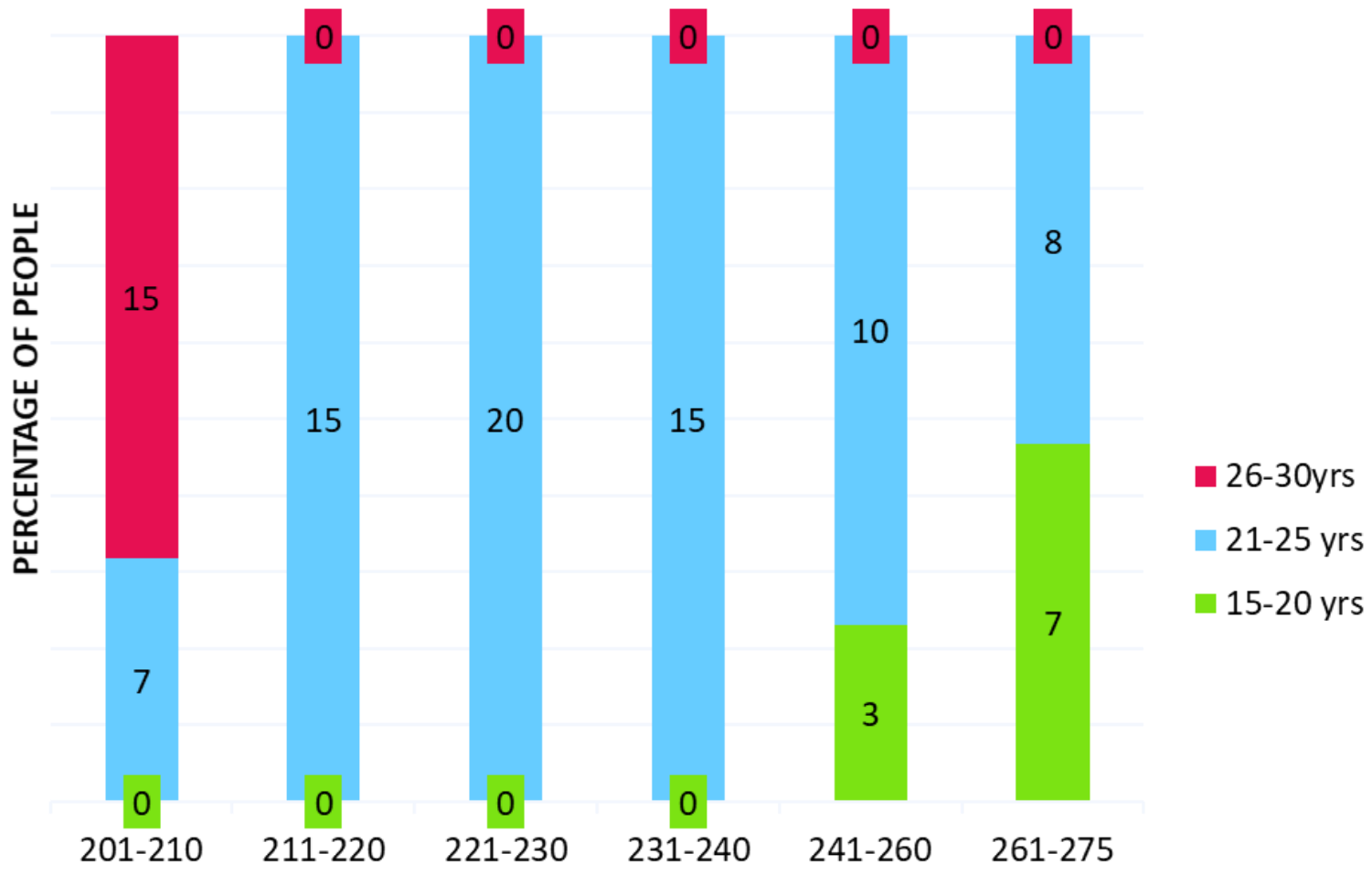

TITRE LEVELS (IU/L)

Such decreasing titres with increase in age could be due to the quality and handling of the vaccine used, the use of a one-dose vaccination schedule, primary or secondary vaccine failure and the waning immunity after vaccination.

Low levels of antibody concentrations in women of fertile age observed in the present study may also affect the quality of measles protection of unvaccinated newborns and infants. Infant protection is provided via transplacental transmission of antibodies in case that the mother has been vaccinated or had measles during life. From this point of view, it is possible to pose a question about the level of protection against measles in children of mothers, who have low seropositivity levels or low antibody concentrations in the serum (14).

All these findings suggest a possible gap in protection of population against measles. A possible confirmation of such is the measles outbreaks, which occurred in many regions of India recently among young adults (5).

The results of the present study have certain limitations as the study was conducted among urban educated youth, who may not be representative of rest of India.

In our study, a significant fraction of the participants with history of immunization 
continue to remain susceptible. Identifying the susceptible population is an important step towards preventing and controlling outbreaks of measles. Eliminating these pockets of susceptible young adults would complement national efforts to control and eliminate measles in future.

Putting a step forward, the Government of India has initiated massive Measles-Rubella Vaccination Campaign in a phased manner in 2017, targeting children aged between 9 months and 15 years, with the aim to eliminate measles by 2020 (20).

Country specific data is needed to ascertain periodicity of such campaigns, which would be based on accumulation of susceptible individuals in a community.

In conclusion, serological monitoring of susceptibility to vaccine preventable diseases like Measles, Mumps and Rubella is an essential tool to monitor immune status in general population. Susceptibility of young adults to measles, as indicated by our study, reinforces the need for multicentric serosurveillance and booster vaccination against these vaccine preventable diseases.

\section{Acknowledgement}

We wish to express our deep sense of gratitude to the technical staff and faculty of Department of Microbiology, Osmania General Hospital for their valuable support and guidance to carry out this study.

\section{References}

1. Bose AS, Jafari H, Sosler S, Narula AP, Kulkarni VM, Ramamurty N, et al., Case based measles surveillance in Pune: evidence to guide current and future measles control and elimination efforts in India. PLoS One 2014; 9: e108786.
2. Choe YJ, Jee Y, Oh MD, Lee JK. Measles elimination activities in the Western Pacific Region: experience from the Republic of Korea. J Korean Med Sci 2015; 30: S115-21.

3. 3) Davidkin I, Valle M. Vaccine-induced measles virus antibodies after two doses of combined measles, mumps and rubella vaccine: a 12-year follow-up in two cohorts. Vaccine. 1998; 16(20): 2052 \pm 7. PMID: 979606.

4. 4) Davidkin I, Jokinen S, Broman M, Leinikki P, Peltola $H$. Persistence of measles, mumps, and rubella antibodies in an MMR-vaccinated cohort: a 20-year follow-up. J Infect Dis 2008; 197: 950_6.7.

5. 5) Prevalence of Measles, Mumps, Rubella and Varicella Susceptibility among Health Science Students in a University in India. G Arunkumar, PhD1, K E Vandana, MD2, and Nalini Sathiakumar, Am J Ind Med. 2013 January; 56(1): 58-64.

6. 6) Global Eradication of Measles: Are We Poised? Kulkarni RD, Ajantha GS, Kiran AR, Pravinchandra KR. Global eradication of measles: Are we poised? Indian J Med Microbiol 2017; 35:10-6.

7. 7) Seroprevalence of measles and natural rubella antibodies among children in Bangui, Central African Republic Manirakiza et al., BMC Public Health 2011, 11:327.

8. 8) Mossong J, Muller CP. Modelling measles re-emergence as a result of waning of immunity in vaccinated populations. Vaccine 2003; 21:4597-603.

9. 9) Park K. Epidemiology of communicable diseases. In: K Park (ed.), Textbook of preventive and social medicine, 21st ed. Jabalpur, India: Banarsidas Bhanot Publishers; 2011

10. 10) Paunio M, Hedman K, Davidkin I, Valle M, Heinonen OP, Leinikki P, et al., Secondary measles vaccine failures identified by measurement of IgG avidity: high occurrence among teenagers 
vaccinated at a young age. Epidemiol Infect 2000; 124:263-71.

11. 11) Poland GA, Jacobson RM. Failure to reach the goal of measles elimination. Apparent paradox of measles infections in immunized persons. Arch Intern Med 1994; 154:1815-20.

12. 12) Measles in India: Challenges \& recent developments. Saurabh RamBihariLal Shrivastava. Infection ecology and epidemiology 2015,5:27784http://dx.doi.org/10.3402/iee.v5.27784.

13. 13) Simons E, Ferrari $M$, Fricks J, Wannemuehler K, Anand A, Burton A, et al., Assessment of the 2010 global measles mortality reduction goal: Results from a model of surveillance data. Lancet 2012; 379: $2173-8$.

14. 14) Smetana J, Chlibek R, Hanovcova I, Sosovickova R, Smetanova L, Gal P, et al., Decreasing seroprevalence of Measles antibodies after vaccination - possible gap in measles protection in adults in the Czech Republic. PLoS One 2017; 12: e0170257.

15. 15)Sugerman DE, Barskey AE, Delea MG, Ortega-Sanchez IR, Bi D, Ralston KJ, et al., Measles outbreak in a highly vaccinated population, San Diego, 2008: role of the intentionally under vaccinated. Pediatrics 2010; 125:747-55.

16. 16) Immune response to second dose of MMR vaccine in Indian children.Sunil Gomber, Shilpa Khanna Arora, Shukla Das, and V. G. Ramachandran. IJMR 2011 Sep; 134(3): 302-306.

17. 17) Swati Bajaj, Pranjal Bobdey1, Neha Singh2Bajaj S, Bobdey $\mathrm{P}$, Singh N. Measles outbreak in adults: A changing epidemiological pattern. Med J DY Patil Univ 2017; 10:447-52.

18. 18) Trmal J, Kupcova J, Dvorakova L, Vaculikova D., Limberkova R, Slajova I et al., Measles re-emerging in the Usti Region. Epidemiol Mikrobiol Imunol.

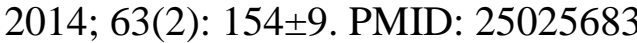

19. 19) Vaidya SR. Commitment of measles elimination by 2020: Challenges in India. Indian Pediatr 2015; 52:103-6.

20. 20) Measles-Rubella Supplementary Immunization Activity Readiness Assessment - India, 2017-2018.Vandana Gurnani,; Pradeep Haldar,; Sudhir Khanal,; Morbidity and Mortality Weekly Report (https://www.cdc.gov/mmwr).

\section{How to cite this article:}

Prashanthi, L., N. Sridevi, Dara Saranya and Sashikala Reddy, P. 2019. Seroprevalence of Measles Antibodies among Young Adults in a Tertiary Care Hospital, India. Int.J.Curr.Microbiol.App.Sci. 8(06): 960-967. doi: https://doi.org/10.20546/ijcmas.2019.806.116 